\title{
New challenges of urothelial carcinoma
}

\section{Opinion}

Urothelial carcinoma (UC) of the bladder is a major cause of morbidity and mortality worldwide, causing an estimated 150,000 deaths per year. ${ }^{1}$ Although the treatment for UC has improved over the last several decades, diagnostic techniques have progressed more slowly. Cystoscopy is still considered the best method for diagnosing UC, but it is invasive, uncomfortable, and can only detect approximately $90 \%$ of lesions. On the other side, urine cytology has the advantages of being a simple, cost-effective, and noninvasive test, while has shown a wide range of sensitivity (11\%-76\%) depending on the specimen type, clinical setting and grade of urothelial tumors. Because cystoscopies are invasive and cytology has poor sensitivity, a non invasive, highly sensitive and specific markers have been sought as alternatives to cystoscopy and cytology for the detection and surveillance of bladder cancer. Urine markers have been evaluated in all clinical scenarios, but often they are not routinely utilized. The limitations of established bladder cancer biomarkers requires us to identify better molecular parameters that could be clinically useful for diagnosis and prognosis, in particular, for the high-risk patient groups that are usually at high risk of progression, recurrence and metastasis. The efficacy of immunotherapy in non-muscle invasive urothelial carcinoma of the bladder was first established in 1976 with Bacillus Calmette-Guerin, but no immunotherapy has been approved for the treatment of advanced disease.

Over time, an improved understanding of immunobiology uncovered the importance of immune checkpoints in facilitating tumor escape, leading to the development of multiple novel therapeutics targeting the CTLA-4 (cytotoxic T-lymphocyte-associated protein 4, CD152) and PD-1/PD-L1 (programmed cell death protein 1, CD279; programmed death-ligand 1, CD274) immune checkpoints. Once the immune checkpoints have been blocked, the equilibrium between the autoimmunity and immune tolerance will be affected. ${ }^{2}$ In particular, melanoma, renal cell carcinoma (RCC), and non-small cell lung cancer (NSCLC) have demonstrated durable responses to immune checkpoint inhibition. In the tumor microenvironment, PD-1 and its ligand PD-L1 perform a vital role in tumor progression and survival by escaping tumor neutralizing immune surveillance. It has been found that PD-1 is expressed on a variety of immune cells, such as monocytes, T cells, B cells, dendritic cells, and tumor-infiltrating lymphocytes (TILs). However, PDL-1 is expressed in tumor cells and antigen presenting cells (APCs), and the engagement of PDL1 with PD-1 of T cell creates T cell dysfunction, exhaustion, neutralization, and interleukin-10 (IL-10) production in a tumor mass. ${ }^{3}$

In 2016, the FDA approved using Atezolizumab for treating patients who have advanced or metastatic urothelial carcinoma in which their diseases worsen during or after using platinum-containing chemotherapy. Atezolizumab was approved based on a multi-center single-arm trial that had 310 patients. It was found that Atezolizumab
Volume 2 Issue 5 - 2017

\author{
Patrizia Straccia, Francesco Pierconti \\ Division of Anatomic Pathology and Histology, Catholic \\ University of Sacred Heart, Italy
}

Correspondence: Patrizia Straccia, Division of Anatomic Pathology and Histology, Catholic University of Sacred Heart, Foundation "Agostino Gemelli" University Hospital, Largo Francesco Vito I, 00168 Rome, Italy, Fax +39-06-30I5-7008, Tel +39-06-30I5-4433, Email stracciapatrizia@libero.it

Received: December 14, 2017 | Published: December 21, 2017

showed good activity as immune checkpoint inhibitors. It was noted that as the expression of PD-L1 increases, the response increases. ${ }^{4}$ Currently, the prognostic value of PD-L1 IC expression is unknown, with conflicting reports in the literature, although it does not appear to be associated with validated adverse risk factors. Ultimately, immunotherapy, and immune checkpoint inhibition in particular, has led to a paradigm shift in oncology, requiring development of new radiographic response criteria and altering expectations for durable responses with therapy. The promising efficacy of these agents combined with an increasing understanding of the complicated interactions between the immune system and tumor foster optimism for progress in biomarker development in this field.

\section{Acknowledgements}

None.

\section{Conflicts of interest}

The authors declare that are no competing interests.

\section{References}

1. Jemal A, Bray F, Center MM, et al. Global cancer statistics. CA Cancer J Clin. 2011;61(2):69-90.

2. Alsaab HO, Sau S, Alzhrani R, et al. PD-1 and PD-L1 checkpoint signaling inhibition for cancer immunotherapy: mechanism, combinations, and clinical outcome. Front Pharmacol. 2017;8:561.

3. Sun Z, Fourcade J, Pagliano O, et al. IL10 and PD-1 cooperate to limit the activity of tumor-specific CD8+T cells. Cancer Res. 2015;75(8):1635-1644.

4. Rosenberg JE, Hoffman-Censits J, Powles T, et al. Atezolizumab in patients with locally advanced and metastatic urothelial carcinoma who have progressed following treatment with platinum-based chemotherapy: a single-arm, multicentre, phase 2 trial. Lancet. 2016;387(10031):1909-1920. 